\title{
Laboreal
}

Volume 8 No2 | 2012

A antropotecnologia, ferramenta ou engodo?

\section{Innovación organizacional : sistemas de actividad, construcción de redes y prácticas asociadas a la adopción de tecnologías de gestión en la administración pública chilena}

Inovação organizacional : sistemas de atividade, construção de redes e práticas associadas à adoção de tecnologias de gestão na administração pública chilena Innovation organisationnelle: systèmes d'activité, construction de réseaux et pratiques associées à l'adoption de technologies de gestion dans l'administration publique au Chili

Organizational innovation: activity systems, creating networks and practices associated with the adoption of management technologies in the Chilean public administration

\section{Gloria Zavala}

\section{OpenEdition}

\section{Journals}

\section{Edición electrónica}

URL: http://journals.openedition.org/laboreal/6806

DOI: $10.4000 /$ laboreal.6806

ISSN: 1646-5237

Editor

Universidade do Porto

Referencia electrónica

Gloria Zavala «Innovación organizacional : sistemas de actividad, construcción de redes y prácticas asociadas a la adopción de tecnologías de gestión en la administración pública chilena », Laboreal [En línea], Volume 8 N² | 2012, Publicado el 01 diciembre 2012, consultado el 24 septiembre 2020. URL http://journals.openedition.org/laboreal/6806 ; DOI : https://doi.org/10.4000/laboreal.6806

Este documento fue generado automáticamente el 24 septiembre 2020

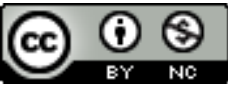

Laboreal está licenciado com uma Licença Creative Commons - Atribuição-NãoComercial 4.0 Internacional. 


\section{Innovación organizacional :} sistemas de actividad, construcción de redes y prácticas asociadas a la adopción de tecnologías de gestión en la administración pública chilena

Inovação organizacional : sistemas de atividade, construção de redes e práticas associadas à adoção de tecnologias de gestão na administração pública chilena Innovation organisationnelle: systèmes d'activité, construction de réseaux et pratiques associées à l'adoption de technologies de gestion dans l'administration publique au Chili

Organizational innovation: activity systems, creating networks and practices associated with the adoption of management technologies in the Chilean public administration

Gloria Zavala

\section{REFERENCIA}

Zavala, G. (2012). Innovación organizacional : sistemas de actividad, construcción de redes y prácticas asociadas a la adopción de tecnologías de gestión en la administración pública chilena. Tesis de Doctorado, Facultad de Ciencias Sociales, Universidad de Chile, Santiago/Chile. 


\section{NOTA DEL EDITOR}

Manuscrito recibido en : Septiembre/2012

Aceptado tras peritaje : Noviembre/2012

\section{Introducción : cambios en la gestión pública}

1 Se realizó un estudio de caso longitudinal en el Fondo de Inversión Social (FOSIS), organismo dependiente del Ministerio de Planificación del Gobierno de Chile, con la finalidad de estudiar procesos de adopción de tecnologías de gestión. El caso se construyó considerando los años 2008 a 2010, en torno a la implementación de un sistema informático de gestión de recursos humanos (SíGestiona) y al levantamiento de competencias y construcción de nuevos perfiles de cargo.

2 En los últimos años las organizaciones públicas chilenas han iniciado un proceso de modernización de sus sistemas de gestión (Ramírez, 2002; Waissbluth \& Inostroza, 2007 ; Waissbluth, 2008). Esta mirada viene de la mano con los planteamientos de un grupo no sistemático de soluciones a la burocracia tradicional, llamado Nueva Gestión Pública (NGP). Y que, a pesar de las críticas, sigue manteniéndose como un pilar en la orientación hacia una mayor eficiencia de las organizaciones del ámbito público (Waissbluth \& Inostroza, 2007).

\section{Problema}

3 Es dentro de esta mirada, que la adopción de innovaciones a nivel de la gestión ha alcanzado gran relevancia (Damanpour, 1991; 1996; Goya, 2002 ; Ramírez, 2002 ; Falivene \& Silva, 2005 ; Bastidas \& Moreno, 2007 ; Damanpour \& Wichnevsky, 2006 ; Damanpour \& Schneider, 2008).

4 Díaz (2010) sugiere que la inclusión de diversos paradigmas de gestión, ha incentivado formas de construcción organizacional con las siguientes características (Díaz, 2010, pp. 21-22) : Incorporación de "sistemas tecnológicos y organizacionales (...) con fuertes discontinuidades en cuanto a su filiación histórica (...) ; fragmentación funcional, social y cultural (...) ; disociación del nexo al interior de la cadena misión, visión, estrategia, procesos, estructura (...)".

5 Lo anterior, dificultaría el surgimiento de representaciones basadas en actividad funcional compartida, requisito para la flexibilidad orgánica que supone la NGP.

Considerando esta problemática, un análisis de la literatura a nivel latinoamericano y nacional, no da cuenta de mayores desarrollos en torno a las dinámicas internas que acompañan a procesos de adopción de innovaciones de gestión, considerando su secuencialidad temporal (Rainieri, 1998; Goya, 2002 ; Waissbluth \& Inostroza, 2006, 2007 ; Waissbluth, 2008).

\section{Objetivo y Marco de Análisis Conceptual}

El estudio apuntó a analizar los procesos de adopción de nuevas tecnologías de gestión en la Subdirección de Personas (SDP) del Fondo de Inversión Social (FOSIS), una 
organización de la administración pública chilena, desde un enfoque de sistemas de actividad y redes.

En base a esto, para la realización del análisis se diferenciaron los siguientes puntos :

1. Propósitos : construcción de propósitos a partir de los discursos de los miembros de la SDP.

2. Sistemas de actividad (Cole \& Engeström, 2001; Engeström, 1987; Engeström, 2003; Engeström \& Escalante, 2001). Implicando puntos de conflicto y solución dentro y entre los sistemas de actividad, ciclos de aprendizaje y desarrollo de competencias, integrando aspectos de las perspectivas de Cognición Distribuida (Hutchins, 1995; Salomon, 1993) y Acción Situada (Suchman, 1987, 2007).

3. Competencias, aprendizaje (Díaz, 2000, 2008), marco tecnológico y mecanismos de cierre de los grupos sociales relevantes (Pinch \& Bijker, 1984).

4. Prácticas de Construcción de redes : entre sistemas de actividad y desde la Teoría del ActorRed (Callon, 1986).

\section{Marco Metodológico}

9 Para alcanzar estos objetivos, se llevó a cabo un estudio de caso cualitativo, descriptivo y longitudinal.

10 Los datos para la construcción del estudio, fueron producidos a través de observación, análisis documental y entrevistas abiertas semi directivas (Ortí, 1992) a los miembros de la SDP, y cubren el período de 2008 a 2010.

11 Se utilizó análisis de discurso y análisis de contenido cualitativo.

\section{Resultados}

12 A nivel del contexto institucional, marco tecnológico y grupos sociales relevantes en torno a la adopción tecnológica, se identificaron 3 tipos de propósitos :

1. Propósito tradicional-normativo : cumplimiento de normas de la Administración Pública.

2. Propósito técnico-instrumental : búsqueda de la eficiencia y la eficacia en la gestión.

3. Propósito administrativo-político : mejora de la imagen interna de la SDP.

13 La organización pública se estructura principalmente desde un propósito tradicionalnormativo, en todas sus unidades, aunque con distintos grados de priorización, ya que en cuanto su existencia y permanencia en el tiempo sólo se ve resguardada a partir de éste. En este sentido, por ejemplo, la informatización se relaciona con la puesta en marcha de fuertes inversiones asociadas a convenios de desempeño.

Las construcción de redes se da a partir de procesos políticos orientados por diversos propósitos, observándose una amplia motivación por grupos principalmente profesionales (en contraste con técnicos y administrativos) por el cumplimiento de propósitos instrumentales, sin embargo, es la lógica burocrática, tradicional-normativa, la única que mantiene finalmente el imperativo de continuar con el uso de las nuevas tecnologías, principalmente cuando éstas se quedan sin portavoz.

15 En este sentido, las expectativas de mejora varían ampliamente cuando se establece un contraste con lo que realmente los artefactos pueden aportar en el trabajo cotidiano, generándose ajustes ad-hoc, con la intención de integrar de alguna forma las nuevas tecnologías, sin salirse de un marco de representaciones comunes de la actividad, 
implicando un esfuerzo de constante negociación socio-técnica por parte de los miembros de la organización.

Las posibilidades de aprendizaje en este contexto, se ven limitadas, debido a la falta de adecuación de los artefactos a las formas de hacer el trabajo, generándose ciclos de solución de problemas contingentes, orientados al cumplimiento de las tareas y a su consiguiente permanencia dentro de la lógica organizacional (considerando principalmente que la coordinación de base es burocrática), sin permitir espacio a la innovación.

\section{Conclusiones}

17 La utilidad de los instrumentos de gestión tanto informáticos como no informáticos, como en el caso de las competencias, se encuentra en que logren constituirse objetos límite, a la vez que puntos de paso obligado, artefactos estabilizados. Esto hace referencia a la construcción de una intersubjetividad, implicando la consideración de la acción como situada, en un escenario que hace sentido a las variaciones del contexto en torno a cómo se articula la actividad.

En consecuencia, se plantea que los límites de un artefacto tecnológico nunca son sólo físicos, son conceptuales, prácticos y políticos. Dentro de una negociación constante de significados nunca estables, nunca no ambivalentes, nunca claros, tanto en términos de las interpretaciones asociadas al artefacto, al proceso y a los grupos participantes.

\section{BIBLIOGRAFÍA}

Bastidas, E., \& Moreno, Z. (2007). El cuadro de mando integral en la gestión de las organizaciones del sector público : Universiad Centroccidental Lisandro Alvarado. Compendium, 10 (018), 5-20. Consultado en octubre, 07, 2010 de http://redalyc.uaemex.mx/pdf/880/ 88001802.pdf

Callon, M. (1986). Some elements of a sociology of translation: domestication of the scallops and the fishermen at St Brieuc Bay. In John. Law (Ed.), Power, action and belief: a new sociology of knowledge? (pp. 196-223). London: Routledge.

Cole, M., \& Engeström, Y. (2001). A cultural-historical approach to distributed cognition. In Gavriel Salomon (Ed.), Distributed Cognitions: Psychological and Educational Considerations (pp. 1-46). New York: Cambridge University Press.

Damanpour, F. (1991). Organizational innovation: a meta-analysis of effects of determinants and moderators. Academy of Management Journal, 34 (3), 555-590.

Damanpour, F. (1996). Organizational complexity and innovation: developing and testing multiple contingency models. Management Science, 42 (5), 693-716.

Damanpour, F., \& Schneider, M. (2008). Characteristics of innovation and innovation adoption in public organizations: assessing the role of managers. Journal of Public Administration Research and Theory, 19 (3), 495-522. 
Damanpour, F., \& Wischnevsky, J. D. (2006). Research on innovation in organizations: Distinguishing innovation-generating from innovation-adopting organizations. Journal of Engineering and Technology Management, 23 (4), 269-291.

Díaz, C. (2000). Impacto Organizacional y en las Personas de las Implantaciones Tecnológicas : dos casos de referencia en Chile. In Actas ESOCITE, s/p, Sao Paulo.

Díaz, C. (2008). Actividad, contexto organizacional y competencias. Revista Psicología Organizacional Humana (UAI), 2, 53-68.

Díaz, C. (2010). Tensiones y proyecciones en la modernización de la gestión pública : discusión desde una perspectiva histórico-cultural. Psicoperspectivas, 9 (1), 7-28.

Engeström, Y. (1987). Learning by expanding: an activity theoretical approach to developmental research. Helsinki: Orienta-Konsultit Oy.

Engeström, Y. (2003). Activity theory and individual and social transformation. In Yrjö Engeström, Reijo Miettinen \& Raija-Leena Punamäki (Eds.), Perspectives on activity theory (pp. 19-38). New York: Cambridge University Press.

Engeström, Y., \& Escalante, V. (2001). Mundane tool or object of affection? The rise and fall of the Postal Buddy. In B. A. Nardi (Ed.), Context and Consciousness. Activity theory and human-computer interaction (pp. 325-373). Cambridge: The MIT Press.

Falivene, G., \& Silva, G. (2005). Formación, cambio tecnológico e innovación en organizaciones públicas. X Congreso Internacional del CLAD sobre la Reforma del Estado y de la Administración Pública, Santiago, Chile. Consultado en julio, 03, 2010 de http://www.sgp.gov.ar/contenidos/ag/paginas/ ponencias/docs/clad_x/

Formacion_cambio_tecnologico_innovacion_organizaciones_publicas.pdf

Goya, F. (2002). Innovación en la gestión pública chilena : dos enfoques alternativos. VII Congreso Internacional del CLAD sobre la Reforma del Estado y de la Administración Pública, Lisboa, Portugal. Consultado en julio, 03, 2008 de http://unpan1.un.org/intradoc/groups/ public/documents/ CLAD/clad0043606.pdf

Hutchins, E. (1995). Cognition in the wild. Cambridge: The MIT Press.

Ortí, A. (1992). La apertura y el enfoque cualitativo o estructural : la entrevista abierta y la discusión de grupo. In Manuel García Ferrando, Jesús Ibañez \& Francisco Alvira (Comps.), El análisis de la realidad social : métodos y técnicas de investigación (pp. 189-221). Madrid: Alianza Universidad Textos.

Pinch, T., \& Bijker, W. (1984). The social construction of facts and artefacts: or how the sociology of science and the sociology of technology might benefit each other. Social Studies of Science, 14, 399-441.

Rainirei, A. (1998). Creencias y cambio organizacional en los sectores público y privado. Estudios Públicos, 70 (otoño), 201-229.

Ramírez, Á. (2002). Innovación en la gestión pública : lecciones, aprendizajes y reflexiones a partir de la experiencia chilena. VII Congreso Internacional del CLAD sobre la Reforma del Estado y de la Administración Pública, Lisboa, Portugal. Consultado en septiembre, 01, 2008 de http:// unpan1.un.org/intradoc/groups/public/documents/CLAD/clad0043607.pdf

Salomon, G. (1993). No distribution without individuals' cognition: a dynamic interactional view. In Gavriel Salomon (Ed.), Distributed Cognitions: Psychological and Educational Considerations (111-138). Ney York: Cambridge University Press. 
Suchman, L. (1987). Plans and situated actions: the problem of human-machine communication. New York: Cambridge University Press.

Suchman, L. (2007). Human-machine reconfigurations. Plans and situated actions ( $2^{\circ} \mathrm{Ed}$.). New York : Cambridge University Press.

Waissbluth, M. (2008). Gestión del cambio en el sector público. Comparative and General Pharmacology, Santiago. Consultado en junio, 14, 2010 de http://old.dii.uchile.cl/boletin/ 20090331/_contenido/publicaciones/1/ Gestiooon_del_Cambio_en_el_Sector_Puuublico,_Mario_Waissbluth.pdf

Waissbluth, M., \& Inostroza, J. (2006). La reforma del Estado en Chile 1990-2005. De la confrontación al consenso. Barcelona : PUBLIC, Boletín del Instituto de Gobernanza y Dirección Pública de ESADE. Consultado en junio, 14, 2010 de http://www.esade.edu/public

Waissbluth, M., \& Inostroza, J. (2007). Globalización y reforma del Estado en Chile. Iberoamericana. Nordic Journal of Latinoamerican and Caribbean Studies, XXXVII (1), 285-310.

\section{AUTOR}

\section{GLORIA ZAVALA}

Universidad de Chile - Facultad de Ciencias Sociales, Departamento de Psicología, Av. Capitán Ignacio Carrera Pinto, nº 1045, Santiago de Chile

gzavala@u.uchile.cl 